\title{
Non-Specific Symptoms As A Prodrome Of Immune- Related Adverse Events In Patients With Non-Small Cell Lung Cancer Receiving Nivolumab: A Consecutive Analysis Of 200 Patients
}

Ryoko Inaba Higashiyama

National Cancer Center Hospital

Hidehito Horinouchi ( $\nabla$ hhorinou@ncc.go.jp )

National Cancer Center Hospital

Aya Kuchiba

Kanagawa University

Yuji Matsumoto

National Cancer Center Hospital

Shuji Murakami

Kanagawa Cancer Center

Yasushi Goto

National Cancer Center Hospital

Shintaro Kanda

Shinshu University

Yutaka Fujiwara

Aichi Cancer Center

Noboru Yamamoto

National Cancer Center Hospital

Yuichiro Ohe

National Cancer Center Hospital

\section{Research Article}

Keywords: Non-small cell carcinoma, Immune checkpoint inhibitors, nivolumab, immune-related adverse events,prodrome, and signal symptom

Posted Date: February 24th, 2022

DOI: https://doi.org/10.21203/rs.3.rs-1360096/v1

License: (9) (7) This work is licensed under a Creative Commons Attribution 4.0 International License. Read Full License 


\section{Abstract}

Introduction: Immune checkpoint blockade therapy is the standard treatment for metastatic or refractory nonsmall cell lung cancer (NSCLC). however, it is associated with immune-related adverse events (irAEs). irAEs are sometimes fatal, however, an efficient method for early irAEs detection has not been developed because their onset timing varies. We examined the significance of non-specific symptoms as a prodrome of irAEs in patients with NSCLC.

Methods: We reviewed consecutive patients who received nivolumab at a dosage of $3 \mathrm{mg} / \mathrm{kg}$ every 2 weeks for metastatic NSCLC between December 2015 and August 2017. Patient demographics, irAEs and signal symptoms were recorded. Non-specific symptoms (fever and fatigue) occurred 7 days or earlier before the onset of irAEs were considered signal symptoms. For statistical analyses, the association between irAEs and clinical information, including signal symptoms, was evaluated using Fisher's exact test and logistic regression.

Results: A total of 200 patients received nivolumab; 131 (65.5\%) were male, their median age was 63 years (range, 30-83), $174(87.0 \%)$ had performance status of 0-1. Signal symptoms occurred in $38(19.0 \%)$ of the 77 patients (38.5\%) who experienced irAEs, and were positively associated with the occurrence of irAEs $(P<0.01)$. Multivariate analysis showed that the occurrence of irAEs was significantly higher in patients with PS 0-1 (odds ratio [OR] 7.01; 95\% confidence intervals [Cl], 1.69-29.13) and patients experienced signal symptoms (OR 17.30; $95 \% \mathrm{Cl}, 6.51-45.99)$.

Conclusions: The occurrence of signal symptoms could be used in the early detection and management of irAEs in patients during immune checkpoint blockade therapy.

\section{Introduction}

Lung cancer remains the leading cause of cancer death globally. Immune checkpoint inhibitors, including nivolumab, have been established as a standard treatment for metastatic or refractory non-small cell lung cancer (NSCLC). Adverse events associated with immune checkpoint inhibitors, especially immune-related adverse events (irAEs), are associated with fatal outcomes and severe decline in organ function and quality of life [1-4]. Adequate diagnosis and management of irAEs are the major issues in identifying the appropriate immune checkpoint inhibitor to be used. Several guidelines and reviews regarding irAEs have been published [5.6]. However, an efficient method for the early detection of irAEs has not been developed, mainly because of different onset timings of irAEs and a wide range of organ systems that can be affected. Moreover, there is little information in the existing guidelines about the prediction and early diagnosis of irAEs. Therefore, we investigated the potential of non-specific prodromal symptoms, such as fever and fatigue, for the early detection of irAEs. Our findings could have clinical significance in the early detection and treatment of NSCLC.

\section{Materials And Methods}

We conducted a retrospective analysis of adult patients (aged $\geq 18$ years) who were cytologically or histologically diagnosed with advanced/metastatic (i.e., stage IIIc/IV) NSCLC and received nivolumab at a dosage of $3 \mathrm{mg} / \mathrm{kg}$ every 2 weeks at the National Cancer Center Hospital (NCCH), Japan, between December 2015 to August 2017; we observed the patients until August 2018 or death. The study was performed as per the 
$\mathrm{NCCH}$ institutional review board-approved protocol (No. 2015 - 355). All patients with malignant thoracic tumors diagnosed and treated at the Department of Thoracic Oncology, National Cancer Center Hospital who consented to the analysis were included in the analysis, and patients who did not consent to the analysis were excluded.

We reviewed the medical records of these patients and collected the following data regarding their pretreatment characteristics: demographics, efficacy and safety of nivolumab, information about irAEs, and signal symptoms. The patients were followed up every 1 to 2 weeks at the outpatient clinic. Signal symptoms were defined as non-specific symptoms (fever and fatigue) that were not present at the time of nivolumab initiation and were present at least 7 days prior to the diagnosis of irAEs.

Medical staff, especially nurses at outpatient clinics, interviewed the patients using a predetermined questionnaire, independent of the physician. The questionnaire consisted of 25 questions that could be answered with a yes or no. The questionnaire was based on the following criteria items. Items are as follows: cough, shortness of breath, dyspnea, itching, rash, skin color change, loss of appetite, diarrhea, bloating, abdominal pain, nausea, joint pain, muscle pain, palpitation, hyperhidrosis, headache, depression, edema, general malaise, jaundice of the conjunctiva bulbar, dry mouth, urine output increase, fever, and blurred vision. Regular systemic work-ups including chest X-ray, chest and abdominal computer tomography (CT), and brain CT or magnetic resonance imaging (MRI) were performed almost regularly every 3 months in the outpatient clinic.

The diagnosis of irAEs was first made by the physician in charge and recorded in the medical records. In this study, two specialists independently reviewed the medical records to confirm whether or not these were irAEs. The CTCAE version 4.0 was used to report adverse events.

The Fisher exact test was used to evaluate the association between the presence or absence of irAEs and the presence or absence of signal symptoms. The multivariate logistic regression model was used to estimate odds ratios (ORs), and 95\% confidence intervals ( $\mathrm{Cls}$ ) were used to estimate the association between irAEs and factors related to patient characteristics. The following potential factors were investigated in the logistic regression model: age, sex, Eastern Cooperative Oncology Group (ECOG) performance status (0-1 vs 2-4), smoking history (past or current vs. never), tumor histology (non-squamous vs. squamous), driver gene alteration (positive vs. negative or unknown), year of treatment initiation (2015-2016 vs 2017 or later), and signal symptoms (presence vs. absence). P-value $<0.05$ was considered to indicate statistical significance. All analyses were performed using STATA ver. 12.0 (Stata, College Station, TX, USA).

\section{Results}

Two-hundred patients with NSCLC received nivolumab at NCCH, Japan, between December 2015 and August 2017 (Figure 1). The baseline patient characteristics are shown in Supplemental Table1. All patients received at least one complete cycle of nivolumab, the median age of the patients was 63 years (range, 30-83 years), 131 patients (65.5\%) were male, $26(13 \%)$ had a PS of 2 or more, 144 (72\%) were past or current smokers, and 40 (20\%) had driver gene alterations, mainly epidermal growth factor receptor (EGFR) mutations. Forty-nine (24.5\%) patients had lung squamous NSCLC, 151 (75.5\%) had non-squamous NSCLC, 130 (65\%) had adenocarcinoma, 6 (3\%) had large cell neuro endocrine carcinoma (LCNEC), 3 (1.3\%) had pleomorphic carcinoma, and 12 (6\%) had others. The median observation period from the start of administration of nivolumab was 275 days (minimum 12, maximum 1017). 
IrAEs appeared in 77 patients (38.5\%). In patients with irAEs, the median time from the start of administration of nivolumab to the onset of irAEs was 29 days (0-518 days). There were 20 cases of Grade 1, 29 cases of Grade 2, 22 cases of Grade 3, 4 cases of Grade 4 and 2 of grade 5 irAEs. Thyroid dysfunction, being the most common irAE, was found in 19 cases (9.5\%), followed by 16 cases (8\%) of dermatitis, 15 (7.5\%) of pneumonitis, $12(7 \%)$ of colitis, 4 of adrenal dysfunction (2\%), 4 of liver dysfunction (2\%), 1 of acute renal dysfunction 1 cases (0.5\%), 1 of autoimmune myocardial damage (0.5\%), 1 of autoimmune cystitis (0.5\%), 1 of autoimmune pancreatitis $(0.5 \%)$, and 1 of uveitis $(0.5 \%)$. The most common irAE of grade 3 or higher was pneumonitis, which occurred in 11 patients (Supplemental Table 2).

Signal symptoms appeared in 46 of the 200 patients treated with nivolumab: fever and fatigue were observed in 21 (10.5\%) and 31 (15.5\%) cases, respectively, and simultaneous occurrence of fever and fatigue was observed in 6 cases. In patients who presented with signal symptoms, the median duration from the start of nivolumab administration to the onset of signal symptoms was 29 days (range, 0-518 days), and the median duration from the appearance of signal symptoms to the onset of irAEs was 28 days (range, 7-259 days).

Signal symptoms were observed in 38 cases in the group with irAEs and 8 cases in the group without irAEs. (Table 1). Among the patients who experienced signal symptoms, $82.6 \%$ had irAEs, and among those who did not, $25.3 \%$ had irAEs (OR 14.0, 95\% Cl: 6.10-32.04).

To minimize the impact of cancer-related death as a competing risk, we examined the association between the presence or absence of signal symptoms and irAEs in 84 patients who were alive at least 1 year after the start of nivolumab administration (Figure 2). We analyzed any correspondence between signal symptoms and irAEs but found no specific association (Table 2). A similar trend was confirmed in analyses conducted with different follow-up periods to minimize competing risks (Figure 2, Supplemental Table2 and 3).

Multivariable logistic regression analysis showed that PS and signal symptoms were significantly associated with the incidence of irAEs (OR for PS 7.01, 95\% Cl 1.69-29.13; OR for signal symptom 17.30, 95\% Cl, 6.5145.99) (Table 3).

\section{Discussions}

This is the first study to report that non-specific signal symptoms can help in the early detection of irAEs. We observed that signal symptoms occurred at a median duration of 25.5 days before the onset of irAEs. It was confirmed that the frequency of subsequent irAEs was significantly higher in patients who experienced signal symptoms than in those who did not (82.6\% vs. $25.3 \%)$. Early detection of irAEs could be achieved by focusing on non-specific signal symptoms during the induction phase of immune checkpoint inhibitors.

In this study, a few non-specific symptoms were screened using a predetermined questionnaire. The odds ratio for subsequent irAEs in patients with signal symptoms was 9.74 (95\% Cl, 2.74-34.63). It is reported that 58$69 \%$ of patients experience the occurrence of some type of irAEs with the administration of immune checkpoint inhibitors [1-4]. Multiple guidelines for irAE management have been published, including guidelines by the American Society of Clinical Oncology and by the National Comprehensive Cancer Network. These guidelines focus on the administration of immunosuppressive agents and hormone replacement therapy[5]. They recommend prompt treatment intervention for irAEs to avoid discontinuation of immune checkpoint inhibitors 
[6-8]. Furthermore, these guidelines emphasize the importance of early detection of irAEs. Based on the current analysis, it might be possible to select patients at high risk for irAEs by identifying signal symptoms in advance (median time of 28 days).

The development of signal symptoms may be related to several cytokines that are produced in association with irAEs. Valpione et al. reported a positive correlation between low blood interleukin (IL)- 6 levels before treatment and ipilimumab-induced irAEs [9]. Another study reported significantly elevated blood IL-6 levels in a population of patients who experienced irAEs with the administration of anti-PD-1 antibody [10]. Cytokines such as IL-6 that are produced via the same mechanism as irAEs, may cause symptoms such as fever, fatigue, and headache before the onset of irAEs. Recently, early increase in inflammatory cytokine levels in the presence of a PD-L1 checkpoint inhibitor was shown to be predictive of the efficacy of the inhibitor in patients with NSCLC [11]. Our study is consistent with this report when combined with studies showing that early onset irAEs are associated with the efficacy of nivolumab in NSCLC ${ }^{12,13}$.

Nevertheless, this study has several limitations. First, owing to the retrospective nature of the study, there could be a possibility of bias in the data analyzed for signal symptoms and irAEs. However, we believe we have minimized recall bias by analyzing information from a predetermined questionnaire that was answered by all patients who received immune checkpoint inhibitors before outpatient visits. In addition, the questionnaire was completed mainly by nurses, independent of the physician. Second, the observation period varied from patient to patient. Patients who have been administered immune checkpoint inhibitors for a longer period may be more likely to develop irAEs. To determine the effect of observation period on the occurrence of irAEs, we compared the incidence of irAEs in both patients with relatively long and short observation periods. The timing of nivolumab initiation was not associated with irAE development in the logistic regression analysis. Although the patient population was analyzed according to whether the patients were observed for more than 1 year from the start of nivolumab administration, signal symptoms were more frequent in patients who developed irAEs regardless of the length of the observation period.

\section{Conclusions}

Signal symptoms (fever and fatigue) are associated with subsequent irAE episodes in patients undergoing nivolumab treatment. By focusing on signal symptoms, prepare for irAEs in advance could be made possible in the clinical setting.

\section{Declarations}

\section{Acknowledgements}

We greatly appreciate the participation of the patients and their families and the assistance of the staff of the Department of Thoracic Oncology of the National Cancer Center Hospital.

\section{Author Contributions}

$\mathrm{RIH}$ and $\mathrm{HH}$ made contributions to the conception and design, acquisition of data, and data analysis. RIH drafted the manuscript. HH made substantial contributions to the study design and revision of the 
manuscript. RIH analyzed and interpreted data and edited the manuscript. All authors critically reviewed the manuscript, and all approved the final version submitted for publication.

\section{Funding}

This research did not receive any specific grant from funding agencies in the public, commercial, or not-for-profit sectors.

\section{Patient consent for publication}

Not required

\section{Ethics approval and consent to participate}

The study was approved by the Institutional Review Board of the National Cancer Center Hospital (No. 2015355).

\section{Provenance and peer review}

No commissioned, extremally peer reviewed.

\section{Data availability}

The datasets generated during the current study are not publicly available due to ethical restrictions, but are available from the corresponding author on reasonable request.

\section{Declaration of interests}

Dr. Higashiyama has nothing to disclose.

Dr. Horinouchi reports grants and personal fees from BMS, grants and personal fees from MSD, grants and personal fees from Chugai, grants and personal fees from Roche, grants and personal fees from Novartis, grants and personal fees from Astra Zeneca, grants from Abbvie, grants from Merck Biophama, grants from Daiichi-Sankyo, grants from Janssen grants from Genomic Health, personal fees from Lilly, personal fees from Kyowa-Kirin, grants and personal fees from Ono, outside the submitted work.

Dr. Kuchiba has nothing to disclose.

Dr. Matsumoto reports grants from National Cancer Center Research and Development Fund, grants from Grantin-Aid for Scientific Research on Innovative Areas, grants from Hitachi, Ltd., personal fees from Olympus, personal fees from AstraZeneca, personal fees

from Novartis, personal fees from COOK, personal fees from AMCO INC, personal fees from Thermo Fisher Scientific, personal fees from Erbe Elektromedizin $\mathrm{GmbH}$, personal fees from Fujifilm, personal fees from Chugai, personal fees from Eli Lilly, outside the submitted work.

Dr. Murakami reports personal fees from AstraZeneca, personal fees from MSD, personal fees from ONO Pharmaceutical, personal fees from Eli Lilly, personal fees from Chugai Pharmaceutical, personal fees from 
Novartis, personal fees from MERCK, outside the submitted work.

Dr. Goto reports grants and personal fees from Eli Lilly, personal fees from Chugai, grants and personal fees from Taiho Pharmaceutical, personal fees from Boehringer Ingelheim, grants and personal fees from Pfizer, grants and personal fees from Novartis, grants and personal fees from AstraZeneca, personal fees from MSD, personal fees from Guardant Health, grants and personal fees from Ono Pharmaceutical, grants from Kyorin, grants and personal fees from Dai-ichi Sankyo, personal fees from Illumina, grants and personal fees from Bristol Myers Squibb, grants from Prefered Network, grants from Abbvie, personal fees from Merck, personal fees from Thermo Fischer, outside the submitted work.

Dr. Kanda has nothing to disclose.

Dr. Fujiwara reports personal fees from Astra Zeneca, personal fees from Daiichi Sankyo, personal fees from ONO Pharmaceutical, personal fees from Otsuka Pharmaceutical, grants and personal fees from Chugai Pharmaceutical, personal fees from Novartis, personal fees from Yakult, grants and personal fees from BristolMyers Squibb, personal fees from Pfizer, personal fees from Takeda, grants from AnHeart Therapeutics, grants and personal fees from Eli Lilly, personal fees from Chiome Bioscience, grants from Amgen, outside the submitted work.

\section{References}

1. Borghaei H, Paz-Ares L, Horn L, et al. Nivolumab versus Docetaxel in Advanced Nonsquamous Non-SmallCell Lung Cancer. N Engl J Med. 2015;373(17):1627-1639.

2. Brahmer J, Reckamp KL, Baas P, et al. Nivolumab versus Docetaxel in Advanced Squamous-Cell Non-SmallCell Lung Cancer. N Engl J Med. 2015;373(2):123-135.

3. Herbst RS, Baas P, Kim D-W, et al. Pembrolizumab versus docetaxel for previously treated, PD-L1-positive, advanced non-small-cell lung cancer (KEYNOTE-010): a randomised controlled trial. The Lancet. 2016;387(10027):1540-1550.

4. Rittmeyer A, Barlesi F, Waterkamp D, et al. Atezolizumab versus docetaxel in patients with previously treated non-small-cell lung cancer (OAK): a phase 3, open-label, multicentre randomised controlled trial. The Lancet. 2017;389(10066):255-265.

5. Julie R. Brahmer, Christina Lacchetti, Bryan J. Schneider, et al. Management of Immune-Related Adverse Events in Patients Treated With Immune Checkpoint Inhibitor Therapy: American Society of Clinical Oncology Clinical Practice Guideline. J Clin Oncol. 2018;36(17):1714-1768.

6. Davies M, Duffield EA. Safety of checkpoint inhibitors for cancer treatment: strategies for patient monitoring and management of immune-mediated adverse events. Immunotargets Ther. 2017; 6:51-71.

7. El Majzoub I, Qdaisat A, Thein KZ, et al. Adverse Effects of Immune Checkpoint Therapy in Cancer Patients Visiting the Emergency Department of a Comprehensive Cancer Center. Ann Emerg Med. 2019;73(1):79-87.

8. Ryder M, Callahan M, Postow MA, Wolchok J, Fagin JA. Endocrine-related adverse events following ipilimumab in patients with advanced melanoma: a comprehensive retrospective review from a single institution. Endocr Relat Cancer. 2014;21(2):371-381. 
9. Valpione S, Pasquali S, Campana LG, et al. Sex and interleukin-6 are prognostic factors for autoimmune toxicity following treatment with anti-CTLA4 blockade. J Transl Med. 2018;16(1):94.

10. Tanaka R, Ichimura Y, Kubota N, et al. Activation of CD8 T cells accelerates anti-PD-1 antibody-induced psoriasis-like dermatitis through IL-6. Commun Biol. 2020;3(1):571.

11. Ozawa Y, Amano Y, Kanata K, et al. Impact of early inflammatory cytokine elevation after commencement of PD-1 inhibitors to predict efficacy in patients with non-small cell lung cancer. Med Oncol. 2019;36(4):33.

12. Haratani K, Hayashi H, Chiba Y, et al. Association of Immune-Related Adverse Events With Nivolumab Efficacy in Non-Small-Cell Lung Cancer. JAMA Oncol. 2018;4(3):374-378.

13. Teraoka S, Fujimoto D, Morimoto T, et al. Early Immune-Related Adverse Events and Association with Outcome in Advanced Non-Small Cell Lung Cancer Patients Treated with Nivolumab: A Prospective Cohort Study. J Thorac Oncol. 2017;12(12):1798-1805.

\section{Tables}

Table1. Relationship between Signal Symptoms and immune-related adverse events (irAEs)

\begin{tabular}{|lllll|}
\hline \multirow{4}{*}{\begin{tabular}{l} 
irAEs \\
Signal \\
\cline { 2 - 5 } symptoms
\end{tabular}} & Yes & No & Total \\
\cline { 2 - 5 } & No & 38 & 8 & 46 \\
\cline { 2 - 5 } & Total & 77 & 115 & 154 \\
\hline
\end{tabular}

Table2. Frequency of immune-related adverse events (irAEs) per signal symptoms

Table 3. Multivariate analysis of immune-related adverse events (irAEs) outcomes $(\mathrm{N}=200)$ 


\begin{tabular}{|c|c|c|c|c|c|c|c|c|c|c|c|c|c|}
\hline & & \multicolumn{2}{|c|}{ irAEs } & \multicolumn{2}{|c|}{$\begin{array}{l}\text { endocrine } \\
\text { disorder }\end{array}$} & \multicolumn{2}{|c|}{ enterocolitis } & \multicolumn{2}{|c|}{ pneumonitis } & \multicolumn{2}{|c|}{ dermatitis } & \multicolumn{2}{|c|}{$\begin{array}{l}\text { liver } \\
\text { dysfunction }\end{array}$} \\
\hline & & ૧ & 口 & 口 & प & 口 & 口 & प & प & प & प & प & 0 \\
\hline \multirow{4}{*}{$\begin{array}{l}\text { Signal } \\
\text { symptom }\end{array}$} & प & 38 & 8 & $14(1)$ & 8 & $1(1)$ & 8 & $7(3)$ & 8 & $6(2)$ & 8 & $1(2)$ & 8 \\
\hline & प & 39 & 115 & 9 & 115 & $10(1)$ & 115 & $6(1)$ & 115 & $8(2)$ & 115 & $1(2)$ & 115 \\
\hline & ORs & \multicolumn{2}{|c|}{14.00} & \multicolumn{2}{|c|}{22.36} & \multicolumn{2}{|l|}{1.44} & \multicolumn{2}{|c|}{16.77} & \multicolumn{2}{|c|}{10.78} & \multicolumn{2}{|c|}{14.38} \\
\hline & & \multicolumn{2}{|c|}{$\begin{array}{l}6.10- \\
32.04\end{array}$} & \multicolumn{2}{|c|}{$7.57-66.28$} & \multicolumn{2}{|c|}{$0.22-10.04$} & \multicolumn{2}{|c|}{$4.74-59.97$} & \multicolumn{2}{|c|}{$3.14-37.57$} & \multicolumn{2}{|c|}{$1.38-120.95$} \\
\hline \multirow[t]{4}{*}{ Fever } & प & 12 & 3 & $4(1)$ & 3 & 0 & 3 & $3(2)$ & 3 & $1(2)$ & 3 & $0(2)$ & 3 \\
\hline & Q & 39 & 115 & 9 & 115 & 10 & 115 & 6 & 115 & 8 & 115 & 1 & 115 \\
\hline & ORs & \multicolumn{2}{|c|}{11.80} & \multicolumn{2}{|c|}{17.04} & \multicolumn{2}{|l|}{ Q } & \multicolumn{2}{|c|}{19.18} & \multicolumn{2}{|l|}{4.79} & \multicolumn{2}{|l|}{ प } \\
\hline & & \multicolumn{2}{|c|}{$\begin{array}{l}3.16- \\
43.99\end{array}$} & \multicolumn{2}{|c|}{$3.29-88.14$} & \multicolumn{2}{|l|}{ Q } & \multicolumn{2}{|c|}{$3.61-104.39$} & \multicolumn{2}{|c|}{$0.63-39.03$} & \multicolumn{2}{|l|}{ प } \\
\hline \multirow[t]{4}{*}{ Fatigue } & प & 20 & 5 & 7 & 5 & $1(1)$ & 5 & $4(1)$ & 5 & 5 & 5 & 1 & 5 \\
\hline & प & 39 & 115 & 9 & 115 & 10 & 115 & 6 & 115 & 8 & 115 & 1 & 115 \\
\hline & ORs & \multicolumn{2}{|c|}{11.80} & \multicolumn{2}{|c|}{17.89} & 2.30 & & 15.3 & & $14.3 \varepsilon$ & & 23.00 & \\
\hline & & $\begin{array}{l}4.1 \\
33 .\end{array}$ & & $4.72-6$ & .85 & $0.24-21$ & 651 & 3.26 & 2.24 & 3.43 & 0.18 & $1.25-$ & 23.41 \\
\hline
\end{tabular}




\begin{tabular}{|c|c|c|c|c|c|c|}
\hline \multicolumn{2}{|l|}{ irAEs } & \multirow{2}{*}{$\begin{array}{l}N \\
46\end{array}$} & \multirow{2}{*}{$\begin{array}{l}\text { Odds Ratio } \\
1\end{array}$} & \multirow[t]{2}{*}{$\mathrm{p}$} & \multicolumn{2}{|c|}{$95 \%$ Conf Interval } \\
\hline \multirow[t]{2}{*}{ Age } & $\geqq 70$ & & & & & \\
\hline & $<70$ & 154 & 1.15 & 0.76 & 0.470 & 2.824 \\
\hline \multirow[t]{2}{*}{ Sex } & female & 69 & 1 & & & \\
\hline & male & 131 & 2.45 & 0.06 & 0.978 & 6.120 \\
\hline \multirow[t]{2}{*}{ ECOG PS } & $2-3$ & 26 & 1 & & & \\
\hline & $0-1$ & 174 & 7.01 & 0.01 & 1.689 & 29.129 \\
\hline \multirow[t]{2}{*}{ Smoking history } & Never smoker & 56 & 1 & & & \\
\hline & Smoker & 144 & 2.74 & 0.05 & 0.986 & 7.611 \\
\hline \multirow[t]{2}{*}{ Tumor histology } & $\mathrm{Sq}$ & 49 & 1 & & & \\
\hline & Non-sq & 151 & 1.46 & 0.39 & 0.616 & 3.475 \\
\hline \multirow[t]{2}{*}{ Driver gene alternation } & Positive & 40 & 1 & & & \\
\hline & Negative & 160 & 2.93 & 0.07 & 0.923 & 9.328 \\
\hline \multirow[t]{2}{*}{ Year of treatment initiation } & 2017- & 25 & 1 & & & \\
\hline & 2015-2016 & 175 & 1.57 & 0.44 & 0.504 & 4.912 \\
\hline \multirow{2}{*}{$\begin{array}{l}\text { Treatment line } \\
\text { of nivolumab }\end{array}$} & 3 or more & 112 & 1 & & & \\
\hline & 2 & 88 & 1.50 & 0.32 & 0.674 & 3.331 \\
\hline \multirow[t]{2}{*}{ Signal symptom } & No & 46 & 1 & & & \\
\hline & Yes & 154 & 17.30 & $<0.01$ & 6.510 & 45.988 \\
\hline
\end{tabular}

ECOG PS; Eastern Cooperative Oncology Group performance status, Sq; squamous cell carcinoma

Figures 


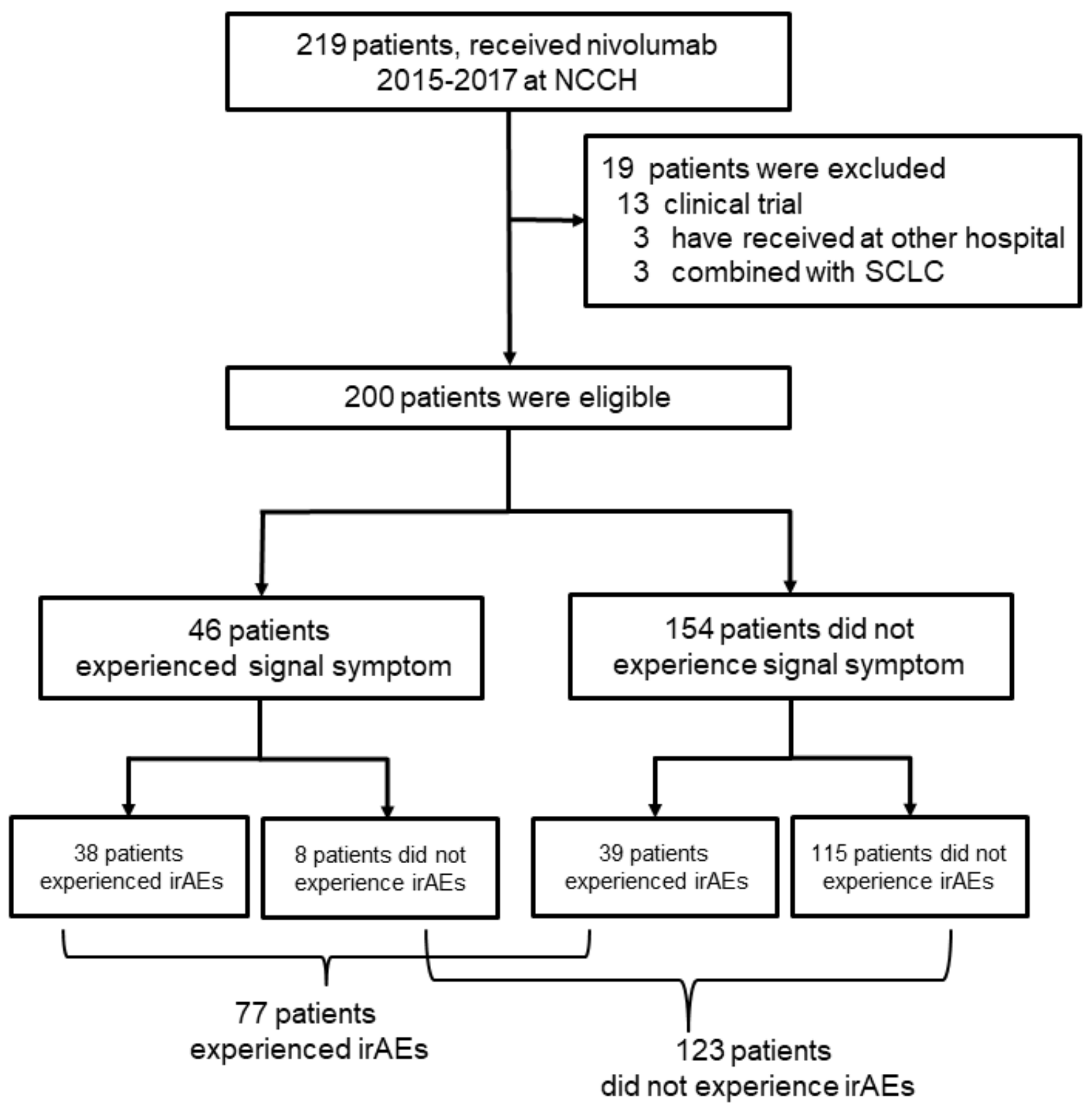

Figure 1

Study flow diagram $(\mathrm{N}=200)$ 
A)

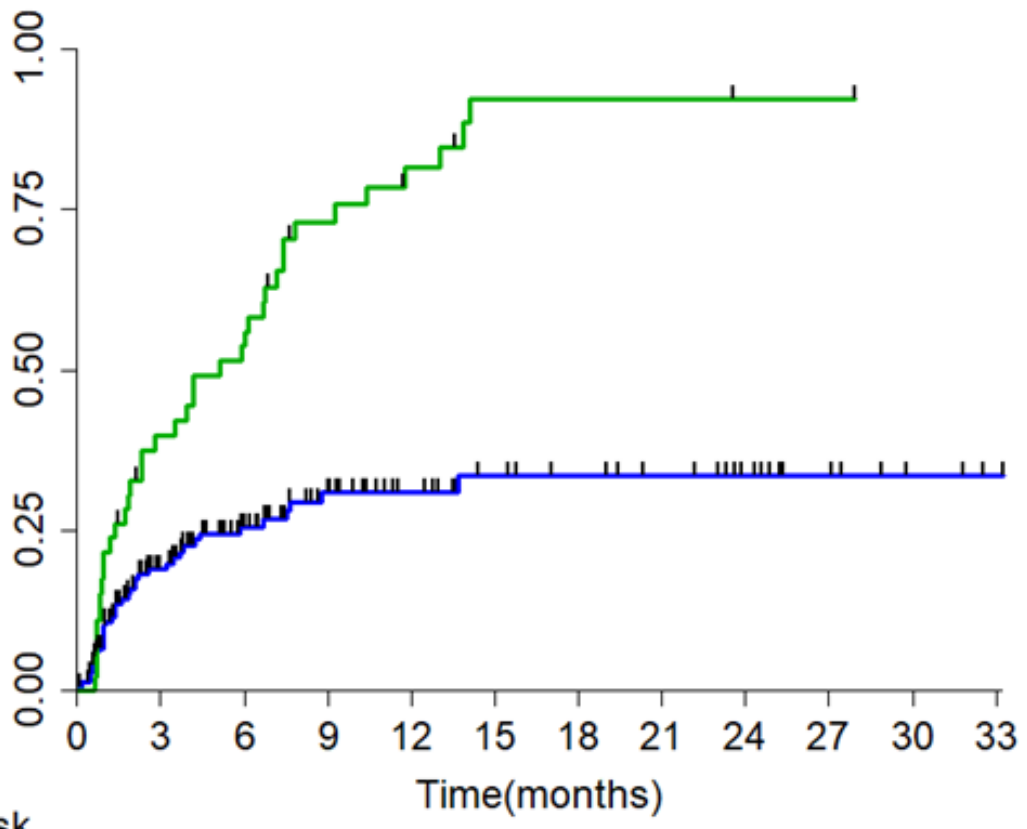

Number at risk

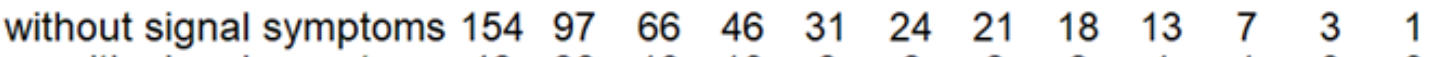

$\begin{array}{lllllllllllll}\text { with signal symptoms } & 46 & 26 & 19 & 10 & 6 & 2 & 2 & 2 & 1 & 1 & 0 & 0\end{array}$

B)

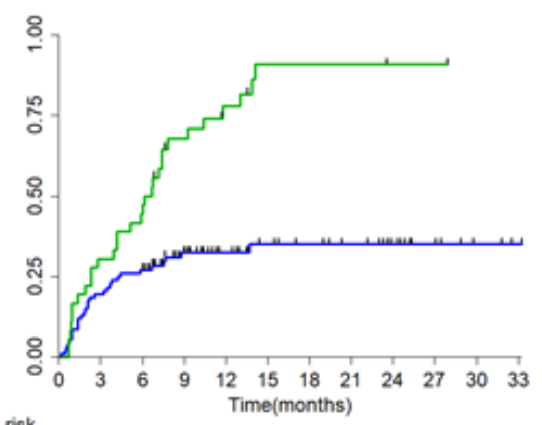

Number at risk

$\begin{array}{lllllllllllll}\text { without signal symptoms } & 92 & 74 & 66 & 46 & 31 & 24 & 21 & 18 & 13 & 7 & 3 & 1\end{array}$

with signal symptoms \begin{tabular}{llllllllllll}
36 & 25 & 19 & 10 & 6 & 2 & 2 & 2 & 1 & 1 & 0 \\
\hline
\end{tabular} with signal symptoms without signal symptoms

with signal symptoms

C)

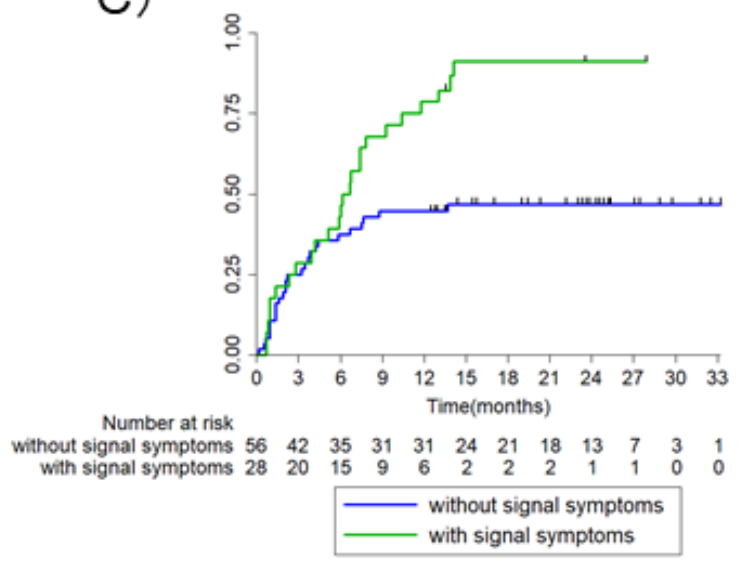

\section{Figure 2}

Immune-related adverse events (irAEs) estimate.

Kaplan-Meier curves for irAEs estimate in (A)All patients, (B) excluded patients who died within a year and (C)excluded patients who died within 6 months 